\title{
Correction to: Anti-inflammatory activity of caffeic acid derivatives isolated from the roots of Salvia miltiorrhiza Bunge
}

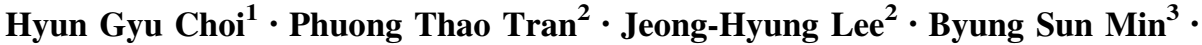 \\ Jeong Ah Kim ${ }^{1}$
}

Published online: 19 November 2018

(C) The Pharmaceutical Society of Korea 2018

\section{Correction to: Arch. Pharm. Res. (2018) 41:64-70 https://doi.org/10.1007/s12272-017-0983-1}

The author would like to include conflict of interest statement of the online published article. The correct conflict of interest statement should read as:
Conflict of interest The authors declare no conflict of interest.

The original article can be found online at https://doi.org/10.1007/s12272-017-0983-1.

Jeong Ah Kim

jkim6923@knu.ac.kr

1 College of Pharmacy, Research Institute of Pharmaceutical

Sciences, Kyungpook National University, Taegu 41566,

Republic of Korea

2 College of Natural Sciences, Kangwon National University, Chuncheon 24341, Republic of Korea

3 College of Pharmacy, Drug Research and Development Center, Daegu Catholic University, Gyeongsan 38430, Republic of Korea 